\title{
A Research of Guangyuan Qianfoya Grotto Statuary Digitization 3D Model Manifestation
}

\author{
Kai Luo, Dengming Gao \\ Neijiang Normal University Zhang Daqian School of Fine Arts \\ hunter2011@foxmail.com
}

Keywords: Guangyuan Qiaofoya Grotto, Digitization, 3D model

\begin{abstract}
Guangyuan Qianfoya Grotto is one of the most valuable natural resources in China. It is an important constituent of Chinese material cultural resources and is a kind of people's direct expression of beautiful life, art appreciation and religious belief in history. It remains an important project about how to protect and inherit grotto art in sustainable development strategy of current natural cultural resources. This paper analyzes the cultural and artistic connotation of Guangyuan Qianfoya Grotto in digital form, summarizes and concludes grotto resources and further studies the feasibility of grotto art digitization 3D model manifestation, thereby making some tentative exploration in protection and sustainable development of promoting grotto art.
\end{abstract}

\section{Research significance of Guangyuan Qianfoya grotto statuary digitization 3D model}

China has a long historical standing, a vast territory and abundant natural and cultural resources. Guangyuan Qianfoya grotto statuary has various forms and unique styles and remains classic inheritance of Chinese cultural resources in the past dynasties. Most of the Guangyuan Qianfoya grotto clusters are chiseled with the mountains. They have vivid and natural forms and far-reaching artistic conceptions. Buddhism statue sanctuary has unique styles and features, beautiful sceneries as well as unique appreciation value, art value and research value. Though some grottos are weathered naturally or destroyed by humans, with the promotion of economic culture, governments and organizations at all levels have made large quantities of work, listing grotto culture as local natural and cultural heritage for protection. In digital image age, 3D model statue and digital renovation and other data image development research of grottos are of important significance to the development of Chinese natural and cultural resources.

At present, characters, recording, video and other forms are utilized in Chinese natural resources protection for objective, systematic and comprehensive recording as well as the establishment of corresponding archive and data base. Digitization modeling through computers and obtaining and processing information through digitization technology are a new form of protecting and developing natural resources. In this way, natural and cultural heritage can be preserved longer. In addition, digital processing of grotto art is more convenient for Internet presentation and promotion. In the meantime, processing obtained information by computer technology can avoid accidental damage to the grottos and is beneficial to better protection and research of grotto artistic resources.

\section{Research approach of Guangyuan Qianfoya grotto statuary digitization 3D model}

With the development of modern society, all countries have paid increasingly more attention to natural and cultural heritage protection. Digital development of cultural heritage has become an important content for researchers of grotto art. Grotto modeling is artists' observation and experience of life during long-term of artistic practices, and it is formed gradually after analysis, judgment and research of grotto statuary. As historical and cultural heritage, Guangyuan Qianfoya grotto is an important constituent of Chinese humane and natural resources. To create Qianfoya grotto artistic local features, it should absorb nutrients from the long-term infiltrative local culture, cultivate one's taste in the long-standing historical river and utilize new mechanism and form to make the existing natural and cultural resources into boutique. As to 3D model digitization of Guangyuan Qianfoya grotto, besides digital recording and storage of grotto art, cartoon, film and 
television as well as graphic images and computers are needed for processing. From the perspective of working means, there are two means: first is the processing of Guangyuan Qianfoya grottos, which means comprehensive and multichannel data resources access and data organization of grotto art by means of digital technology and equipment. The other is development and propagation of grotto art, which means digital modeling and processing of traditional grotto statuary by means of computer to find characteristics of traditional art and establish corresponding geometric model and then study, present and propagate it after computer processing. All in all, on the basis of inheriting previous grotto art forms, finding digital preservation and manifestation techniques of grottos, designing and developing excellent natural and cultural resources to promote inheritance, research and development of grotto art.

Grotto artistic digitization information acquisition and recording. Grotto artistic digitization information recording means storage, management and presentation of grotto art by traditional means, utilizing specific instruments or advanced digital equipment such as digital camera, video camera and other new resource capture equipment for accurate image, geometrical shape, texture and other information and then storing them into computers. Recording and sharing the information can provide reliable digital materials for digital research and development of grotto art.

Guangyuan Qianfoya grotto statuary data processing. Guangyuan Qianfoya grotto statuary data processing means creation, modification and transformation of grotto statuary by means of modern technology and relative equipment. It includes digital transformation of traditional materials or 3D digital scanning of some natural and cultural heritages and then presentation in 3D way on this basis. Digitization technology can realize perpetual lossless storage and virtual restoration in preservation of cultural heritages such as grotto statuary, mural colors and so on. Besides this, digitization technology has numerous advantages in virtual demonstration, restoration performance and optimized propagation of grotto art.

\section{Formation process of Guangyuan Qianfoya grotto digitization 3D model}

Grotto statuary digitization 3D modeling is a means of research and development of natural resources. Grotto digital model researches may go through processes such as two dimensional graphic plotting, three dimensional modeling, model texture mapping and drawing and so on; after a series of manufacturing processes, the pattern is output from three dimensional software and other rendering procedures. Guangyuan Qianfoya grotto statuary began to be established from the Northern Wei Dynasty and has gone through about 1,500 years of history. There are famous Dayun Cave, Dafo Cave, Lianhu Cave and so on. These delicate grotto statuaries provide importance basic conditions for digital development of the grottos.

As to the application analysis conclusion of current 3-D restoration application, current 3-D digital software is divided into 2 dimensional graphic plotting software, 3-D modeling software, texture detail processing software as well as procedure operation display software and so on. During digital development process, the overall process and thought are largely identical but with minor differences. Therefore, finding lifelike details of digital grottos and rapid manufacturing methods are especially important. One should conduct accurate measurement on the digital modeling grottos and obtain orthophotoquad with correct proportions. Front and side photos of grottos should be treated as modeling input. Under the premise that the concise modeling shape is correct, setting out specific textures according to the structure of the statue and bringing in relative data for comprehensive molding until the shape physique is completed. On the basis of existing model, conducting 3-D patter manifestation, statue texture can get better manifestation.

\section{Guangyuan Qianfoya grotto digitization 3-D model virtual manifestation}

Grotto digitization 3-D model is applied in various ways in data age. Objective manifestation and interaction of Guangyuan Qianfoya grotto is one of the exceptional functions of 3-D model auxiliary virtual reality technologies. In research cases of Guangyuan Qianfoya grottos, virtual reality technology supported by digital 3-D model can solve restrictions in Guangyuan district and 
popularity of grottos and so on. It can establish unique grotto art virtually in computers according to the planning scheme and assist visitors for objective judgment. Virtual planning can conduct 3-D modeling in natural scenes and then integrate the new planned model with 3-D models of natural scenes together for presentation and interaction and so on.

Guangyuan Qianfoya grotto digitization 3-D model network virtual presentation. Grotto art virtual presentation based on network has utilized 3-D model, video, audio, animation, image-text and other means; it takes 3-D model of cultural relics as index, which can present the original appearance of Guangyuan Qianfoya grotto more comprehensively, more vividly and more lively. When users are roaming in the virtual real scenes, they can receive introduction to the sceneries, integrating humanistic knowledge background with grotto art and it is the best way of grotto publicity and education. Visitors can also observe the statue of the domination from any perspective, which renders them most freedom and which is impossible in on-site sceneries. Besides, virtual presentation of grotto art has more flexibility, and there is no real terrain, light and restrictions from other aspects during the presentation process. It can establish shapes of the theme pavilion according to requirements and there is more freedom for the arrangement of relative exhibits of grotto art. Exhibits can be put in the exhibition room without considerations of temperatures and humidity. By use of the infinite communication function of the Internet, users can have direct linkage directly and they can discuss and express their own opinions about some problems of knowledge of grotto art. Virtual presentation of the 3-D model is not only simple publicity presentation, it is to attract participation of the visitors more comprehensively, thereby forming a collision of knowledge and culture so that people's understanding of grotto art civilization can be transformed from passive infusion to active appreciation and study, which is a more comprehensive culture popularization and knowledge exploration.

Guangyuan Qianfoya grotto digitization 3-D model virtual interactive experience. By establishing Guangyuan Qianfoya grotto digitization 3-D model virtual interactive experience system, the public can have experiences of virtual roaming, breaking restrictions of time and space; they can experience evolutions of Qianfoya grotto civilization and experiencing charms of Chinese cultural resources. There are numerous kinds of virtual displays, such as ring 3-D interactive display, touch screen display, ball curtain display and so on. In the market, most of the existing 3-D virtual interactive experiences are conducted by audiences' visual input and they have to wear some relative equipment to achieve better visual feelings. Most of the existing displayed digital museum, 4-D and 5-D movies adopt 3-D mode; computers calculate and generate images that the left and right eyes can see and project and amplify. After audiences wear modified or red and blue glasses or other proofread equipment, 3-D stereopsis that imitate the real world would appear. Combining dynamic seats and atmospheric environment, users can be personally on the scene. This kind of technology is mature relatively, and the cost and input is moderate, which is one of the optimized plans of grotto digital 3-D model. With the development of science and the progress of time, naked eye 3-D vision, i.e., the technology of appreciating 3-D stereopsis without equipment is also advancing towards us. The manifestation and application of grotto art digitization 3-D model will be more comprehensive.

\section{Conclusion}

Guangyuan Qianfoya is excellent Chinese natural and cultural heritage, whose extended development and sustainable development are important content that the society values. Researches and explorations of digital 3-D technology of grotto art is becoming increasingly prevailing. In order to achieve better research achievements, human-induced restoration and computer scanning are integrating gradually. Today, when science is developing increasingly rapidly, research method of the combination of culture and science is a new mode of grotto art research. Therefore, conducting 3-D modeling and digital statue virtual development on grotto art and other natural resources by means of digital technology can allow more people feel the charm of Guangyuan Qianfoya grotto artistic resources, enhance people's understanding of grotto art and promote prosperity and development of Chinese cultural resources. 


\section{Acknowledgements}

Fund program: major project of Sichuan Province Education Department Inheritance of Grotto Art__ A Research of Digital Renovation of Guangdong Qianfoya Grotto and Statue Art Development, project number: 13SA0002)

\section{References}

[1] Fan Jinshi. Application of Digital Technique in Preservation and Presentation of Dunhuang Caves. Dunhuang Research, 2009(6)

[2] Ma Zhongping. Overall Development of Cultural Undertaking and Culture Industry. Qiushi. 2005 (23)

[3] Luo Jingyan, Zhu Lili. A Research of Tianjin Folk Arts Intangible Cultural Heritage Protection. Lantai World. 2013 (20)

[4] Wan Jianmin. A Research of Country Historical Sites based on Digitization. Agricultural Archaeology. 2011 (01)

[5] Ke Changqing, Feng Xuezhi, Gu Guoqin. 3-D Information Restoration of Dunhuang Frescoes Digital Image. Nanjing University Journal (Natural Science). 2006(06)

[6] A Research of Sichuan Xiaoliangshan Mountain Yi Nationality Bimo Culture Inheritance and Preservation. Lantai World. 2012 (35) 